\title{
Web Service Testing Automation using SoapUI Tool
}

\author{
Meenakshi Garg \\ Department of MCA \\ VESIT \\ Mumbai, India
}

\author{
Niveta Lavhate \\ Department of MCA \\ VESIT \\ Mumbai, India
}

\begin{abstract}
In today's world, web services have become an integral part of web. A web service is a service offered by one device to another device electronically, for communicating with each other over the World Wide Web. The web services can be also act as an API which facilitates various services for devices over network. Today majority of web applications which keep running makes the use of web services and use service-oriented architecture. A service-oriented architecture (SOA) is a way of designing software where services are provided to the other components by application components, through a communication protocol over the network. The testing of these web services should be considered as an important factor now. Testing the web service manually becomes complicated. The solution is test automation! Web services can be tested by using test automation frameworks. SOAPUI is such an open source tool which can be used for testing of web services. SOAPUI provides a variety of testing facilities. This tool provides the web service tester a modern way through which he can save his time and perform testing efficiently.
\end{abstract}

\section{Keywords}

SOAP, SOAPUI, REST, Automation testing, Web Service Testing

\section{INTRODUCTION}

Test automation has become a trend in today's world. Automation tests are more efficient and exact when contrasted with the manual testing. [1] There are various open source tools available for web service testing. SOAPUI is one most commonly used tool for web service testing. It can discover the imperfections which can be easily missed out while manual testing. It can be used for web service and API testing. [2] It can provide instant and accurate results. Testing is a basic necessity of any application since it gives chance to measure the quality of programming. There is no other way to check if the web service is serving its purpose or not. The web service is not used only by a single system; it is used widely by multiple systems. [3] It becomes necessary to test the web services efficiently as they serve a large number of software systems in order to guarantee the reliability. Web services do not have any user interface which becomes another obstacle in testing of web services. [4] Web service testing includes functional testing, regression testing, load/stress testing and monitoring. In any scenario, manual testing requires excessive human efforts and it is prone to fail due to human mistakes. [5], [6] whenever the programming code changes everything needs to be retested. Manually testing it again and again becomes tedious and unreasonable.

\section{REVIEW OF SOAPUI}

The SOAPUI appeared in 2005. The person behind its existence is Ole Lensmar. [7] He was working in Eviware software's as an architect of a huge SOA project and he realized that there is no test tool available for testing agile development process and he decided to develop it. He worked on SOAPUI in his free time and when Ole and his colleagues realized what SOAPUI can do they made it available to other people working with SOA and result was the open source SOAPUI version got released. [7] Eviware Software was acquired by SmartBear later in 2011.The open source SOAPUI tool is licensed under European Union Public License. This tool is downloaded 2,000,000 times since its initial release. SoapUI NG Pro is a commercial version of SAOPUI which focuses on additional functionalities and enhanced productivity. The SOAPUI tool is completely developed using Java platform which makes it cross-platform. It makes use of Swings framework for user interface. IDEA, Eclipse and Net Beans are also supported by SOAPUI at today's date. SOAP, REST web services, JMS, AMF can be tested by using SOAPUI and it can also be used for making HTTP(S) and JDBC calls.

\section{CHOOSING THE SOAPUI TOOL}

There will be a lot of reasons available for choosing SOAPUI tool. SOAPUI is an open source tool which means it is freely and easily available for use by anybody. This tool is also available in a commercial version called SoapUI NG Pro with extra functionalities for mission critical web services. SOAPUI is considered as a standard for API testing in today's world. With this tool, functional testing, load testing, regression testing, performance testing, interoperability testing and many more things are possible. [8] It aims on testing to be easy. For instance, we can just right click on functional test and run it as a load test, it is that simple to use. Web services can be simulated by using this tool. Test recording and later reusing are most convenient features available in SOAPUI. Code stubs from WSDL can be created using it. Even the creation of the REST specifications (WADL) from recorded communication is possible.

\subsection{Platforms supported by SOAPUI}

As the SOAPUI is java based tool, it can be easily run across various operating systems, it has been tested with multiple Windows versions, Mac and multiple Linux dialects. So, the operating system will not be an issue for those who are willing to make use of this tool. The basic requirements are $1.6+$ versions of the JRE (Java Runtime Environment), at least $1 \mathrm{~GB}$ memory and $100 \mathrm{MB}$ of disk space which are again not difficult to be available. [8]

\section{SOAPUI TOOL IMPLEMENTATION}

There is multiple ways for implementing the web services. Two of the most common approaches are -

a) SOAP - (Simple Object Access Protocol)

b) REST-(Representational State Transfer architecture)

XML format is used by SOAP for sending and receiving request and data is platform independent. Exchange of messages between SOAP provider applications and receiving applications happens in the form of SOAP envelopes. [9] 
SOAP messages do not get block by the firewall because it makes use of simple http transport protocol. In case of REST, its architecture runs over HTTP. REST uses simple URL in some cases instead of using XML requests. REST requests are faster and hence used in mobile and web applications. In order to understand SOAPUI, it is necessary to have knowledge about WSDL. WSDL is Web Service Description Language. It is based on XML and used for describing the services provided by the web service. All the operations provided by a particular web service are described in a XML format by WSDL. It also specifies that how the services can be called i.e. what is the value for input and what response we should get for each type of service.

So, in addition with functional testing SOAPUI also provides non-functional testing such as performance and security testing.

\subsection{SOAPUI Features}

\section{Commonly used features of SOAPUI are listed below}

\subsubsection{Functional Testing}

Testers are allowed to write functional API tests using SOAPUI. It has drag and drop feature which makes the script development faster. [10] SOAPUI allows test debugging. It also provides support for data driven tests. It supports more than one environment hence it is not difficult to switch between QA, Production or Dev environment. It supports advanced scripting which makes easy for testers to develop their own custom code in necessary scenarios. Using SOAPUI tester can use a large number of features. Some of them are listed below

\subsubsection{Drag and Drop}

It is the best feature in perspective of new testers for building test automation. [11] It speeds up the script development for test automation.

\subsubsection{Custom Scripts:}

SOAPUI allows custom test script writing for test automation scripts development which is an advance scripting option that provides flexibility for writing the scripts for test automation.

\subsubsection{Environment Switching}

SOAPUI allows testing the web API by switching in multiple environments such as UAT (User Acceptance Testing), QA (Quality Assurance), SIT (System Integration) etc.

\subsubsection{Test Debugging}

SOAPUI helps tester in debugging the test scripts and allows user to write data driven tests. Tester can put multiple check points to check that the test is getting executed as expected or not.

\subsubsection{Robust Tool}

By considering all the above scenarios SOAPUI is a robust testing tool for sure.

\subsubsection{Compliance Testing}

SOAPUI allows tester to validate the compliance tests and automate those test scripts. Compliance testing consists of the tests related to the source and the data authentication by using license and certificates etc. It is also related to various service level agreements.

\subsubsection{Regression Testing}

Regression testing is performed to analyze any malfunctioning happening within the web services after any new change is introduced. It is carried out to find out if new changes are causing any problems in expected working of the web service. [11] Using SOAPUI tool tester can execute the test suits to check if the existing functionalities are working fine.

\subsubsection{Security Testing}

SOAPUI can perform complete vulnerability scan set. SOAPUI ensures authorization and authentication for request and response web service model. Features that SOAPUI supports under Security testing are listed below

\subsubsection{Cross Site Scripting}

It scans as well as detects any exposure related to service parameters in structure message during cross site scripting.

\subsubsection{Securing database}

SOAPUI scans for any potential $1 \mathrm{SQL}$ injection that could cause possible harm to the database. Hence provides the secure database.

\subsubsection{Stack overflow}

SOAPUI can scan and detect large documents and can find out the stack overflow related defects.

\subsubsection{Lifesaving scans}

These scans are done to support unsteady behavior of some APIs. SoapUI NG Provides fuzzy scans, boundary scans etc.

\subsubsection{Load Testing}

SOAPUI can be used to distribute the Load Tests across multiple numbers of LoadUI Agents. It can simulate real world and high volume load testing easily. To capture performance parameters, it provides advanced custom reporting options. End to end system monitoring is also enabled by SOAPUI. The features provided by load testing are:

\subsubsection{LoadUI Agent}

SOAPUI NG has something called LoadUI agents in large number on which load can be distributed and performance analysis, server monitoring and visualizations can be done.

\subsubsection{Monitoring Performance}

SoapUI NG provides advance reporting system for capturing various performance parameters in load testing. Apart from this it also provides performance monitoring for end-to-end system load testing.

\subsection{Supported Protocols/Technologies}

SOAPUI supports most comprehensive set of protocols which is depicted in Fig 1 .

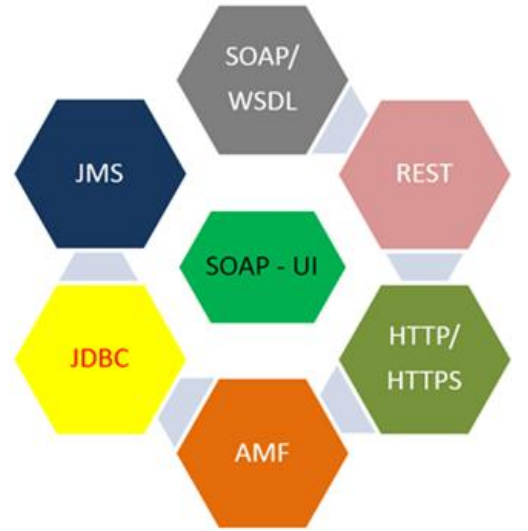

Fig 1: SoapUI Supported Protocols 
SOAP -Simple Object Access Protocol

WSDL - Web Service Description Language

REST - Representational State Transfer

HTTP - Hyper Text Transfer Protocol

HTTPS - Hyper Text Transfer Protocol Secure

AMF - Action Message Format

JDBC - Java Database Connectivity

JMS - Java Messaging Service

\subsection{SOAP-Integration}

SOAP can be integrated with many tools like Maven, Hudson, Junit, Apache Ant and many more. Few of them are described as under -

\subsubsection{Maven}

Apache Maven is a tool for project management that can manage the builds of project, documentation and repository from a central location. SOAPUI tests can b e executed using Maven with simple command.

\subsubsection{Hudson}

Hudson is a java based continuous integration tool which integrates with CVS, Subversion, Git, Perforce and RTC. SOAPUI integrates with Hudson to find out the bugs that any developer commits while developing the software.

\subsubsection{Junit}

SOAPUI test flow can be controlled by Junit testing framework which is used for testing modules.

\subsubsection{ApacheAnt}

It is a command-line tool used for software building. Using SOAPUI's command line Ant automated tests can be executed.

\subsection{SOAPUI Overview}

A brief overview of SOAPUI features is provided here which includes [12] Menu Bar, Navigator, Interface Viewer, Request and Response Panel, Test Suit, Properties Windows and various types of Logs maintained by SOAPUI

\subsection{1Мепи B ar}

The File menu which is depicted in Fig 2 has various options for creating new projects for SOAP or REST requests. It also has options to import existing project. Apart from this it allows to open, close and save projects. [12] The file menu provides us an option for setting global SOAPUI preferences. All the menus in SOAPUI has tool tip text which tells what an option does by hovering over it.

SoapUl 5.3.0
File Project Suite Case Step Iools Desktop $\underline{\text { Help }}$

Fig 2: SOAPUI Menu Bar

The Project menu on the menu bar has various options regarding projects. It allows to add WSDL and WADL (Web Application Description Language) to an existing project. It also provides an option for adding new rest service from URI. This menu allows user to create the SOAP and REST Mock Services. Apart from this it also allows to launch Test Runner, LoadTestRunner, HTTP Monitor and Security Test Runner. Suite, Case and Step menus are specific to a Test Suite, Test case and Test Step respectively. They become active only on selecting the Test suit, Test case or Test Step. Tools menu allows doing required configurations for the project. Finally, the Help menu provides address of helpful resources privacy related terms of use and about the version of software.

\subsubsection{File}

As shown in Fig 3, SOAPUI tool has a visual and handy structure of the File menu. Below the Menu bar there is shortcut for File which allows single click solution for all options present under the File menu. User can directly use these options by clicking on it instead of going into File menu again and again.

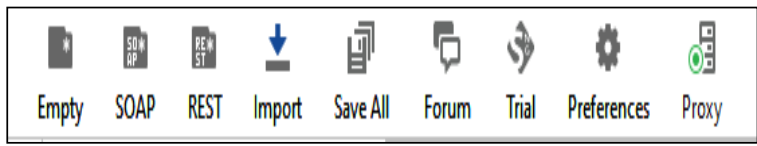

Fig 3: SOAPUI shortcut for File

\subsubsection{Navigator}

The Navigator filed on SOAP UI tool allows navigating across various projects. Fig 4 shows a snapshot of SOAPUI Navigator. Navigator allows switching between the projects and services of the project.

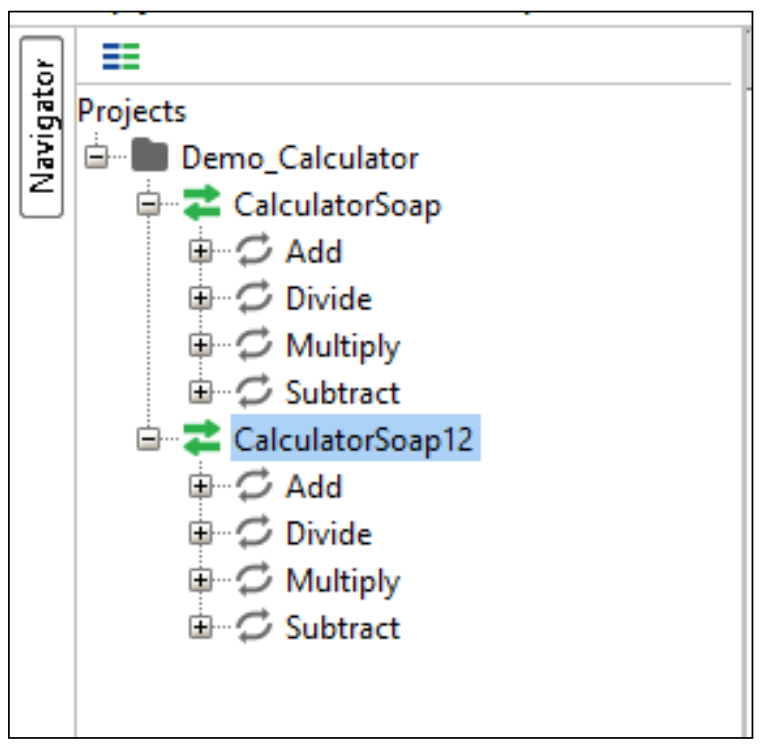

Fig 4: SOAPUI Navigator

\subsubsection{Interface Viewer}

It provides the important information about SOAP/REST service. It also provides details about WSDL, Service endpoints and WSDL content.

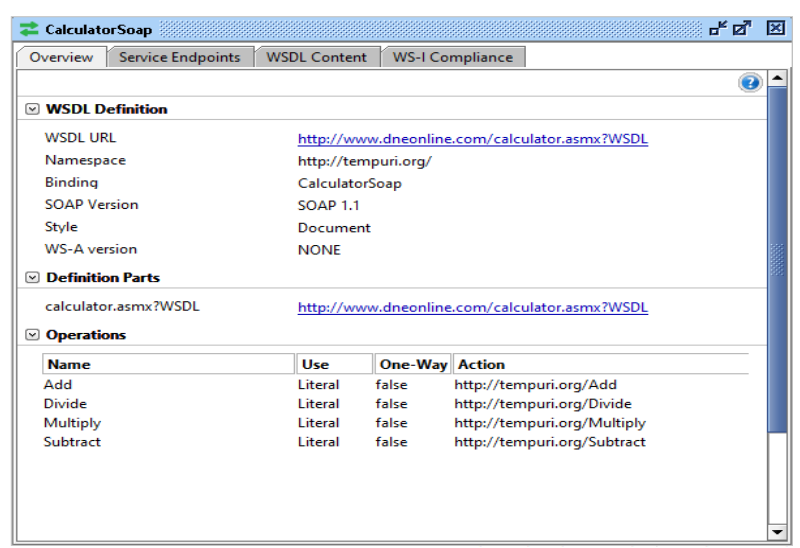

Fig 5: Interface Viewer 
Interface Viewer is depicted in Fig 5. This can be opened by double clicking on the SOAP/REST service.

The Request panel has various options. The first green run button is used for hitting the request. The required input value is given in the inputs fields which initially contains the question mark(?) sign. As soon as the request is hit, the response is shown in the response panel. The response then can be validated. The request and response can be viewed in two formats that are Raw and XML. Both tabs can be used to see the different versions of same request. The bottom line fields on these panels are Authorization, Headers, Attachments, WS-Addressing and WS-Reliable messaging. These fields are used for authentication and other security or header related settings.[11]

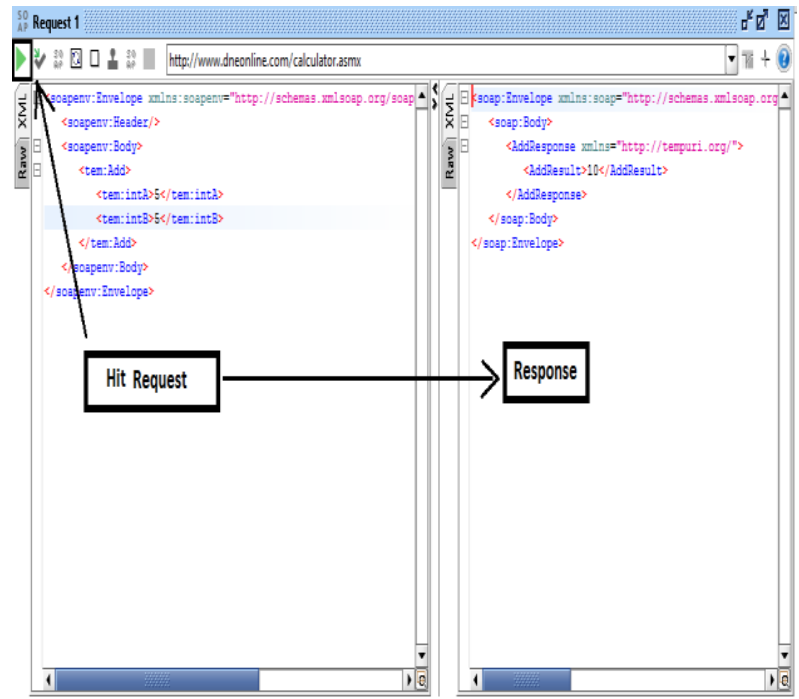

Fig 6: Request and Response Panel

\subsubsection{Test Suit}

The Test Suit in SOAPUI can be created by right clicking on the project. After creating Test Suit, Test cases are added into it. Each test case can contain multiple test steps. The test step can be a SOAP request, REST request, HTTP request, AMF request or JDBC request as shown in the Fig 7.

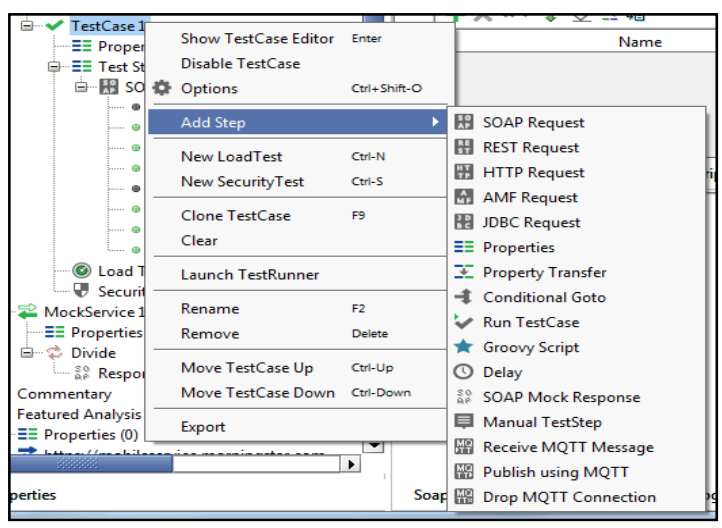

Fig 7: Adding Test step in test case

The major role in test step is played by Assertions. SOAPUI allows user to add assertions of various types. By double clicking on the test step, test step is opened in new window which has a green ' + ' sign and it can be used for adding assertions.

\subsubsection{Assertions}

Assertion means act of affirming or stating something[12] it can be seen as a check point or a validation point. Window for adding Assertion is shown in Fig 7. Assertions have many types like Property Content, Compliance, Status and Standards, Script, SLA, JMS and Security. Each assertion has its description given in SOAPUI. So, the user can go and select the appropriate assertion.[12]

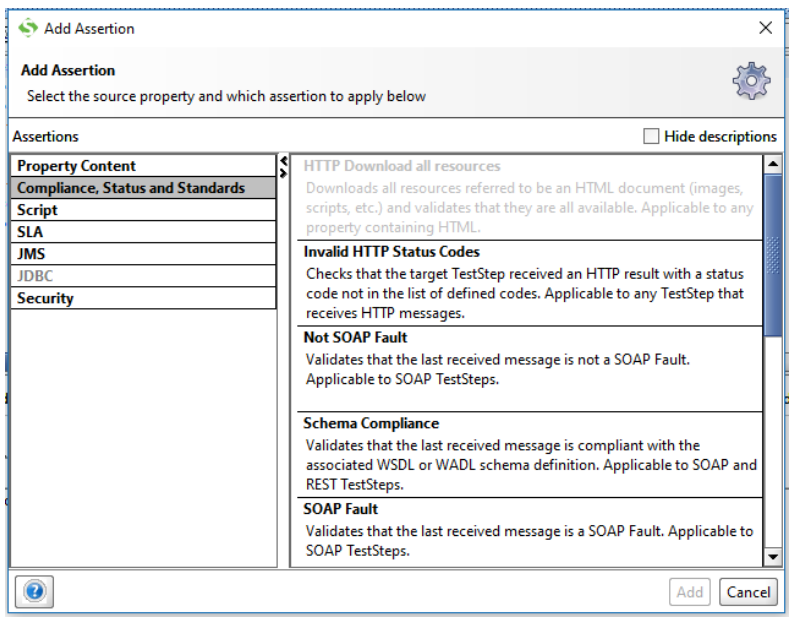

Fig 7: SOAPUI Assertions

\subsubsection{Properties}

At the end of the Navigator there is a tab called Properties. Properties tab is shown in Fig 8. This field provides the details of Name, Description, file address, Resource Root, Cache Definitions, Project password, Script Language and Hermes Configuration.[13]

\begin{tabular}{|l|l|}
\hline \multicolumn{1}{|c|}{ Project Properties } & Custom Properties \\
\hline $\begin{array}{l}\text { Name } \\
\text { Description }\end{array}$ & \multicolumn{1}{c|}{ Value } \\
File \\
Resource Root \\
$\begin{array}{l}\text { Cache Definitions } \\
\text { Project Password } \\
\text { Script Language } \\
\text { Hermes Config }\end{array}$ & true \\
\hline
\end{tabular}

Fig 8: Properties

\subsubsection{Logs}

SOAPUI displays various logs like SOAPUI log, http log, and memory log at the bottom of the tool interface. These logs are depicted in Fig 9, Fig 10 and Fig 11 respectively.

\subsubsection{SoapUI log}

The SoapUI log is used for displaying the information of response from the web server. This information is stored inside the soapui.log file which is stored under the SoapUI installation folder under the 'bin' directory. 
Tue May 30 10:45:04 IST 2017:INFO:Adding plugin from [C:\Users \Nlavhat Tue May 30 10:45:04 IST 2017:ERROR:An error occurred [com.eviware.soap Tue May 30 10:45:04 IST 2017:ERROR:An error occurred [com.eviware.soap Tue May 30 10:45:04 IST 2017:INFO:5 plugins loaded in $1643 \mathrm{~ms}$

Tue May 30 10:45:04 IST 2017:INFO:All plugins loaded

Tue May 30 10:45:05 IST 2017:INFO:Loading workspace from [C:\Users \Nla Tue May 30 10:45:05 IST 2017:INFO:Loaded project from [file:/C:/Users/Nla Tue May 30 10:45:05 IST 2017:INFO:Loaded project from [file:/C:/Users/Nla Tue May 30 10:45:05 IST 2017:INFO:Loaded project from [file:/C:/Users/Nla Tue May 30 10:45:06 IST 2017:INFO:Loaded project from [file:/C:/Users/Nla Tue May 30 10:45:06 IST 2017:INFO:Loaded project from [file:/C:/Users/Nla Tue May 30 10:45:07 IST 2017:INFO:Loaded project from [file:/C:/Users/Nla Tue May 30 10:45:07 IST 2017:INFO:Loaded project from [file:/C:/Users/Nla Tue May 30 10:45:07 IST 2017:INFO:Used java version: 1.7.0_55

Scheduling garbage collection every 60 seconds The cajo server is running on localhost:1198/soapuiIntegration 4

SoapUI log httplog jettylog error log wsrm log memory log

Fig 9: SoapUI Log

\subsubsection{Http Log:}

It is used for displaying the HTTP packet transfer. The information is shown in RAW format in HTTP Log.

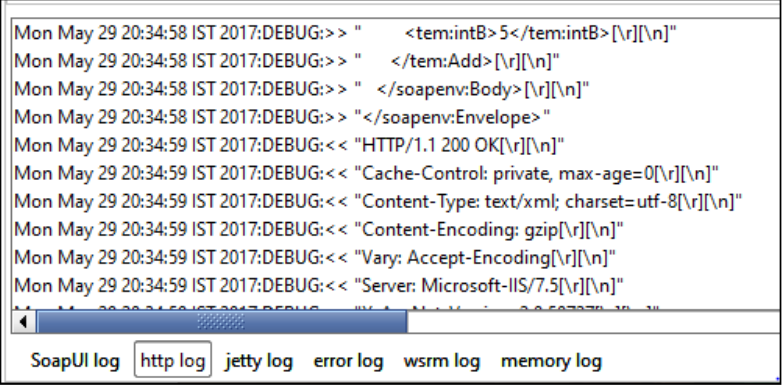

Fig 10: Http Log

\subsubsection{Memory Log}

The memory log monitors the memory consumption and displays it in the form of the charts. When any memory intensive operation is performed this type of log plays an important role.

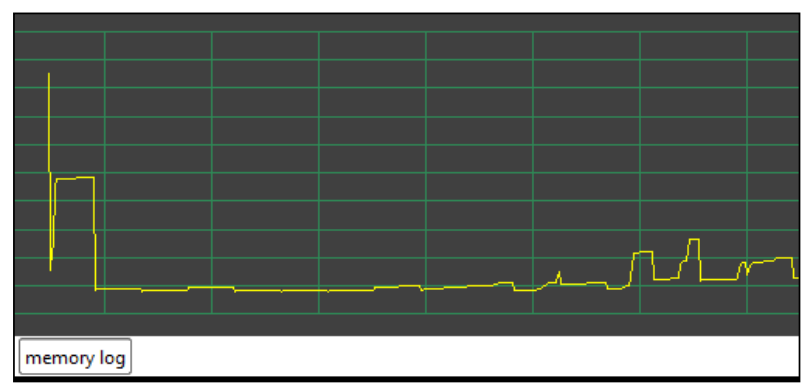

Fig 11: Memory Log

Apart from all these SoapUI also provides the operations of jetty $\log$, error $\log$ and wsrm $\log$ also.

\subsection{SOAPUI NG Pro features}

SoapUI NG Pro has additional features such as more number of views like HTML, JSON, Outline and Overview including basic XML and Raw view tabs. It also has more number of assertions.[14] Its additional features are listed below -
- It provides the data driven testing to save time for checking numerous real-world conditions.

- SoapUI NG Pro manages the multiple environment related configuration at one central location which makes it easy to witch between environments without much overhead.

- It provides test coverage which can be used for positive and negative tests and non-functional tests.

- $\quad$ SoapUI NG Pro provides the API refactoring, API discovery and API monitoring.

- It provides test history, test debugging and result comparison. It gives functionality of Point-andclick testing, Code completion, Global events.

\section{SOAPUI (OPEN-SOURCE) DRAWBACKS}

SOAPUI has got certain drawbacks also . Some of them are listed below -

- SoapUI gives issues when working with JSON response if it contains special characters. So, it is not easy to add assertion on JSON response.

- Work flow testing and Load testing are difficult to perform.

- SoapUI is easy for testing but difficult for documentation.

- It is also observed that the SoapUI customer support response is late.

\section{CONCLUSION}

SoapUI is an open source tool which is easily available for everybody to use. It also has a commercial version with the additional features. Continuous improvement of the SoapUI is going on and making it stronger day by day. As it is an open source tool various intelligent people are contributing towards it and making it more efficient to use. Due to existence of such tool it has become easy to test the web services and web APIs. A lot of time and efforts required by manual testers are saved due to this. SoapUI can be seen as a solution for automation of API testing. Going forward more and more people will start making use of it and it will become an inseparable part of automation testing of web services. Rather we can say that it has already become an integral part of the web service and API testing.

\section{REFERENCES}

[1] Shariq Hussain, Paper on Web Service Testing Tools: A Comparative Study

[2] Ravi Kumar, Research article on A Comparative Study and Analysis of Web Service Testing Tools

[3] Evan Martin, Paper on Automated Robustness Testing of Web Services

[4] Mustafa Bozkurt, Testing Web Services: A Survey

[5] Vinod Patil, Introduction to Testing Web services

[6] Tanuj Wala, A Comparative Study of Web Service Testing Tools

[7] SmartBear Software Further Expands by Acquiring World's Most Widely Used Web Services Testing Tool soapUI https://smartbear.com/news/press-releases/ smartbear-acquiring-web-services-testing-tool/ 
[8] Getting Started With SoapUI https://www.SoapUI.org/getting-started/introduction .html/

[9] Understanding SOAP and REST Basics And Differences https://blog.smartbear.com/apis/understanding-soap-andrest-basics/

[10] Why use SoapUI Pro for Functional Testing? https://www.soapui.org/functional-testing/gettingstarted.html

[11] API Testing Features https://www.soapui.org/opensource/features.html
[12] Introduction for the SoapUI Newbie https://www.soapui.org/getting-started/10-tips-for-thesoapui-beginner.html

[13] Installation and Configuration of SoapUI http://www.softwaretestingclass.com/installation-andconfiguration-of-soap-ui-complete-guide/

[14] Advanced SOAP and REST API Functional Testing with SoapUI NG Pro https://www.soapui.org/professional/ soapui-ng-pro.html 